\title{
Sangrado y transfusión masiva
}

\author{
MARIO CONCHA P. ${ }^{1}$, RADARANIDA GÓMEZ R. ${ }^{2}$
}

1 División de Anestesiología, Hospital Clínico, Escuela de Medicina, Pontificia Universidad Católica de Chile. Santiago de Chile.

2 Residente División de Anestesiología, Hospital Clínico, Escuela de Medicina, Pontificia Universidad Católica de Chile. Santiago de Chile.

\begin{abstract}
The case of a patient that bleed approximatelly 40 lts during a liver and kidney transplant is reported. Strategies directed to maintain hemodynamic and hemostatic conditions are discussed. Elective surgery in patients that may require massive transfusion allow to planify a strategy directed to avoid hypothermia, appearence of metabolic acidosis and coagulopathy. All of these conditions have been related to the appearence and maintenance of bleeding. Maintenance of hemostatic condition appears particularly important requiring a strategy of early administration of blood components directed to preserve an adequate level of clotting factors and platelets. The same considerations must be kept in mind when the requirement of massive transfusion is not expected and the coagulation condition must be preserved or restored.
\end{abstract}

Key words: Massive transfusion, coagulopathy, blood components.

\section{RESUMEN}

Se reporta el caso de un paciente que sangró 40 litros durante un trasplante hepático y renal, discutiéndose las estrategias utilizadas, mantener adecuadas condiciones hemodinámicas y hemostáticas. La cirugía electiva en pacientes que pueden requerir transfusión masiva permite planificar la implementación de medidas dirigidas a prevenir la aparición de hipotermia, acidosis metabólica y coagulopatía, los cuales han sido identificados, relacionados a la aparición y mantención de un estado de mayor sangrado. La mantención de adecuadas condiciones hemostáticas aparece como un factor de gran importancia, requiriendo una estrategia que requiere la precoz administración de hemocomponentes, con la idea de mantener adecuados niveles de factores de coagulación y plaquetas. Estas mismas consideraciones deben tenerse presente cuando el requerimiento de transfusión masiva es imprevisto, y las condiciones de hemostasia deben ser mantenidas o restauradas.

Palabras clave: Transfusión masiva, coagulopatía.

Correspondencia a:

Dr. Mario Concha P.

Marcoleta 367. Santiago - Chile.

Teléfono: 87686899

mconcha@med.puc.cl 


\section{Introducción}

Diversos cuadro médicos u obstétricos, algunos tipos de cirugía o el trauma, pueden presentar sangrado masivo (SM), que requerirán transfusión masiva (TM) y administración de grandes cantidades de fluidos. El manejo de la situación requiere una estrategia que permita reducir las complicaciones asociada a esto. Reportamos un paciente con un sangrado de 40 litros y se discute el manejo.

\section{Caso clínico}

Paciente de 60 años, con antecedente de cirrosis hepática e insuficiencia renal crónica sometido a trasplante hepático y renal. En el pre operatorio destaca MELD 24, INR 1,5, TTPA 42 segundos y plaquetas $42.000 / \mathrm{mm}^{3}$. Como vías venosas se utilizaron 2 introductores $8.5 \mathrm{Fr}$ conectados a calentadores de fluidos. Se utilizaron además frazadas calentadoras de aire para la mitad inferior y superior del cuerpo y cell-saver. Durante la hepatectomía sangra alrededor de 10 litros, detectándose $\mathrm{K}$ hasta $6 \mathrm{mEq} / \mathrm{lt}$, calcio iónico de $2,2 \mathrm{mEq} / \mathrm{lt}$, y hematocrito (Hto) hasta $18 \%$. Se aporta glóbulos rojos (GR), plasma fresco congelado (PFC) y Voluven ${ }^{\circledR}$. Al reperfundir se observa abundante sangrado por distintos sitios, con presión arterial (PA) hasta $30 / 20 \mathrm{mmHg}$ y frecuencia cardíaca (FC) hasta 40 por min con ondas $\mathrm{T}$ altas en el monitor. Se inicia compresión torácica, se administran 0,2 $\mathrm{mg}$ de adrenalina, $1 \mathrm{~g}$ de cloruro de calcio, glucosa e insulina, acelerándose la administración de GR, PFC y Voluven ${ }^{\circledR}$, restableciendo $\mathrm{PA}$ de $95 / 50 \mathrm{mmHg}$ en alrededor de un minuto. Persiste sangrado importante, aportándose coloides y productos sanguíneos para mantener PA alrededor de 100/60 mm Hg. Los exámenes muestran Hto $15 \%, \mathrm{pH} 7,16$, bicarbonato $14,7 \mathrm{mEq} / \mathrm{lt}$, EB -15, lactato $2,2 \mathrm{mEq} /$ lt, Na $140 \mathrm{mEq} / \mathrm{lt}$, K 5,9 mEq/lt, Ca iónico 1,1 $\mathrm{mEq} / \mathrm{lt}$ y glicemia $268 \mathrm{mg} \%$. La temperatura se mantiene sobre $36^{\circ} \mathrm{C}$. Se administran $10 \mathrm{~g}$ de bicarbonato y $7 \mathrm{~g}$ de cloruro de calcio con gradual normalización de estos valores. Conseguida una adecuada hemostasia quirúrgica se administran 10 unidades de plaquetas y se controlan exámenes de coagulación que muestran 45.000/ $\mathrm{mm}^{3}$ plaquetas, INR 1,4 y TTPA 37 segundos.
El trasplante renal transcurre sin incidentes. El balance hídrico final estimó un sangrado de 40 litros, reponiéndose 40,9 litros. De estos, 7,2 lts fueron GR recuperados del cell saver, 7,5 litros de GR de banco (24 unidades), 8,5 litros de PFC (40 unidades), 1,2 lts de plaquetas (20 unidades), ringer lactato 2 lts, Voluven ${ }^{\circledR} 6 \% 12$ lts y 2,5 litros de manitol y suero glucosado. Evoluciona estable en el postoperatorio, extubándose al cuarto día. La creatinina fue $2,6 \mathrm{mg} \%$ al tercer día y $2 \mathrm{mg} \%$ al quinto día.

\section{Discusión}

En un SM la primera y más evidente de las medidas es detener el sangrado. Desafortunadamente esto no siempre es fácil. Mientras se consigue se debe aportar fluidos y hemocomponentes que disminuirán la cantidad y calidad de los factores de coagulación desarrollándose una coagulopatía dilucional, y alterando el balance entre activadores e inhibidores de la coagulación. En algunas situaciones se puede agregar hiperfibrinolisis o, como en el paciente reportado, factores de la patología de base, que deben considerarse al planificar la reposición de volumen. En trauma, el sangrado es la principal causa de las muertes potencialmente evitables ${ }^{1,2}$, siendo la principal causa de ésto el desarrollo de coagulopatía. Esta se produce por las razones mencionadas, agregándose una grave disfunción plaquetaria estableciéndose una condición propia del trauma que es la coagulopatía inducida por trauma (CIT). La alta mortalidada asociada a la TM obliga a buscar una estrategia que permita evitar la aparición de acidosis, hipotermia y coagulopatía ${ }^{3}$, las que perpetuarán el sangrado. Las medidas de protección térmica, calefacción y humedificación de los gases inspirados y calefacción de fluidos asociado a un soporte hemodinámico que asegure una adecuada perfusión tisular resultan fundamentales para controlar los dos primeros. Prevenir la coagulopatía resulta más complejo, requieriendo una estrategia que identifique tempranamente qué pacientes requerirán TM, y el uso precoz de $\mathrm{GR}, \mathrm{PFC}$ y plaquetas ${ }^{4}$. Antecedentes como lesión penetrante, $\mathrm{PA}<100 \mathrm{mmHg}, \mathrm{FC}>120$ al ingreso, $\mathrm{Hb}<7 \mathrm{~g} / \mathrm{dL}$, evidencias de hemoperitoneo, fractura de huesos largos o pelviana, o déficit de base $<-10$, son factores de riesgo de requerir 
$\mathrm{TM}^{5}$. Cirugías electivas vasculares, ortopédicas, trasplante hepático, o complicaciones obstétricas como placenta previa, acretismo placentario o la inercia uterina se asocian también a riesgo de TM. El apoyo hemodinámico requiere una monitorización precisa. La PVC y presión de capilar pulmonar son muy cuestionadas como indicadores del estado del compartimento intravascular ${ }^{6,7}$. La ecocardiografía transesofágica permite visualizar las condiciones de llenado ventricular ${ }^{8}$, pero la disponibilidad del equipo y de operador entrenado limitan su utilización. Lo más útil para guiar la reposición durante la etapa de sangrado activo es la PA, por lo cual una línea arterial debe instalarse rápidamente si no está presente. No hay consenso de la PA y Hto óptimos como objetivo de reanimación. La posibilidad de limitar los problemas derivados de la cantidad de aporte, ha llevado a desarrollar estrategias como la hipotensión permisiva, la que restringe el aporte de volumen buscando obtener PA sistólicas de 80-100 mmHg o PAM de $65 \mathrm{mmHg}^{9}$ los que por períodos cortos podría mantener una perfusión satisfactoria ${ }^{10}$. El lactato y déficit de base permitirán evaluar el impacto del sangrado en la perfusión y la efectividad de la reposición ${ }^{10}$.

Se ha reportado que si la relación PFC:GR se acerca a 1 habría mejor sobrevida, sin embargo, la evidencia que soporta esta afirmación es insuficiente $^{11}$. Más importante que esta relación sería la existencia de protocolos de TM que permitan una precoz reanimación cardiopulmonar y hemostática $^{12}$. En el paciente reportado, considerando la patología hepática y renal, se buscó mantener el volumen intravascular aportando coloides y PFC buscando reducir el efecto dilucional de factores de coagulación y aportando factores que no podrían ser aportados por el hígado del paciente. En caso de sangrado se aportaría precozmente hemocomponentes. Un tratamiento racional de las alteraciones de coagulación requeriría evaluar con exámenes de laboratorio, sin embargo, estos requieren tiempo y no siempre reflejan la real tendencia hemorragípara. La tromboelastografía (TEG) permite evaluar más rápidamente cada etapa de la coagulación, abriendo nuevas posibilidades para guiar de manera más objetiva el manejo de la coagulación en $\mathrm{TM}^{13}$. Coloides y cristaloides pueden mantener condiciones hemodinámicas satisfactorias, pero ambos disminuirán la concentración de plaquetas y factores de coa- gulación. Si bien se ha cuestionado el uso de Voluven ${ }^{\circledR}$ en pacientes críticos, no existe consenso acerca de la validez de estas objeciones, menos aún en casos de recambios de volemia como el reportado ${ }^{14,15}$. En este paciente no se administró fibrinógeno por considerarse que se perdería rápidamente, y dadas las dificultades que puede representar el conseguirlo, se prefirió esperar hasta tener una hemostasia quirúrgica razonable. Sin embargo, debe considerarse que en SM, es el factor que más precozmente alcanza niveles críticos, por lo que debe monitorizarse y administrar 1-5 g cuando los niveles plasmáticos son inferiores a $200 \mathrm{mg} / \mathrm{dl}^{10}$. En Chile no se dispone de fibrinógeno por lo cual debe administrarse como crioprecipitado. El uso precoz de $10-15 \mathrm{ml} / \mathrm{kg}$ de PFC y luego dosis adicionales de acuerdo a resultado de exámenes de coagulación ha sido recomendado en pacientes con $\mathrm{SM}^{10}$. Éste estaría tambien indicado cuando el tiempo parcial de tromboplastina activada (TTPA) o el tiempo de protrombina (TP) son 1,5 el valor normal o después de 10 unidades de GR. En relación a las plaquetas, en pacientes con TM se recomienda mantener sobre $50.000 / \mathrm{mm}^{3}$ y sobre $100.000 \mathrm{~mm}^{3}$ en caso de lesión de sistema nervioso central. Muy probablemente estas recomendaciones de aporte de hemocomponentes se modificarán en la medida que se establezcan protocolos de manejo de TM basado en los resultados de la TEG.

La TM puede producir alteraciones electrolíticas importantes. Factores como la antiguedad de los GR y velocidad de infusión, pueden determinar aumentos importantes de kalemia, especialmente en pacientes con factores de riesgo como insuficiencia renal crónica, o situaciones con gran daño tisular como en trauma. Posteriormente, la restauración de la función de membrana de los GR administrados, y el desarrollo de alcalosis metabólica secundaria a la metabolización de citrato pueden producir hipokalemia. La presencia de acidosis metabólica durante TM es atribuible a hipoperfusión y no a la administración de hemocomponentes. Cristaloides y coloides, por un mecanismo de dilución, y hemocomponentes, por unión del calcio al citrato que estos contienen, pueden producir hipocalcemia.

Finalmente debe mencionarse que una revisión reciente muestra que el cell saver en cirugía cardíaca y ortopédica, reduce los requerimientos de sangre de banco ${ }^{16}$. Esto podría extenderse a 
otras situaciones como la reportada y trauma en la cual se requerirán cantidades importantes de GR.

En resumen, el SM requiere considerar desde el inicio, la mantención de condiciones de perfusión, temperatura, y de factores de coagulación que impidan la perpetuación y agravación de las condiciones circulatorias y hemostáticas.
El caso reportado muestra la importancia de una adecuada preparación. Cuando el SM se presenta de manera imprevista, la pronta implementación de las medidas para tratar de controlarlos y establecer una estrategia de aporte de fluidos y hemocomponentes, resultan fundamentales para un buen resultado.

\section{Referencias}

1. Thorsen K, Ringdal KG, Strand K, Søreide E, Hagemo J, Søreide K. Clinical and cellular effects of hypothermia, acidosis and coagulopathy in major injury. $\mathrm{Br}$ J Surg 2011 Jul;98(7):894-907.

2. Cothren CC, Moore EE, Hedegaard HB, Meng K. Epidemiology of urban trauma deaths: a comprehensive reassessment 10 years later. World J Surg 2007 Jul;31(7):1507-1511.

3. DeLoughery TG. Coagulation defects in trauma patients: etiology, recognition, and therapy. Crit Care Clin 2004 Jan;20(1):13-24.

4. Schöchl H, Maegele M, Solomon C, Görlinger K, Voelckel W. Early and individualized goal-directed therapy for traumainduced coagulopathy. Scand J Trauma Resusc Emerg Med 2012;20(1):15.

5. Nunez TC, Voskresensky IV, Dossett LA, Shinall R, Dutton WD, Cotton BA. Early prediction of massive transfusion in trauma: simple as ABC (assessment of blood consumption)? J Trauma 2009 Feb;66(2):346-352.

6. Marik PE, Baram M, Vahid B. Does central venous pressure predict fluid responsiveness? A systematic review of the literature and the tale of seven mares. Chest 2008 Jul;134(1):172-178.

7. Raper R, Sibbald WJ. Misled by the wedge? The Swan-Ganz catheter and left ventricular preload. Chest 1986 Mar;89(3):427434.

8. American Society of Anesthesiologists and Society of Cardiovascular Anesthesiologists Task Force on Transesophageal Echocardiography. Practice guidelines for perioperative transesophageal echocardiography. An updated report by the American Society of Anesthesiologists and the Society of Cardiovascular Anesthesiologists Task Force on Transesophageal Echocardiography. Anesthesiology 2010 May;112(5):1084-1096.

9. Lier H, Krep H, Schroeder S, Stuber F. Preconditions of hemostasis in trauma: a review. The influence of acidosis, hypocalcemia, anemia, and hypothermia on functional hemostasis in trauma. J Trauma 2008 Oct;65(4):951-960.

10. Rossaint R, Bouillon B, Cerny V, Coats TJ, Duranteau J, Fernández-Mondéjar $\mathrm{E}$, et al. Task Force for Advanced Bleeding Care in Trauma. Management of bleeding following major trauma: an updated European guideline. Crit Care 2010;14(2):R52.

11. Rajasekhar A, Gowing R, Zarychanski R, Arnold DM, Lim W, Crowther MA, et al. Survival of trauma patients after massive red blood cell transfusion using a high or low red blood cell to plasma transfusion ratio. Crit Care Med 2011 Jun;39(6):15071513.

12. Riskin DJ, Tsai TC, Riskin L, Hernandez-Boussard T, Purtill M, Maggio PM, et al. Massive transfusion protocols: the role of aggressive resuscitation versus product ratio in mortality reduction. J Am Coll Surg 2009 Aug;209(2):198-205.

13. Tanaka KA, Bolliger D, Vadlamudi R, Nimmo A. Rotational thromboelastometry (ROTEM)based coagulation management in cardiac surgery and major trauma. J Cardiothorac Vasc Anesth 2012 Dec;26(6):10831093.

14. Myburgh JA, Finfer S, Bellomo R, Billot L, Cass A, Gattas D, et al. CHEST Investigators; Australian and New Zealand Intensive Care Society Clinical Trials Group. Hydroxyethyl starch or saline for fluid resuscitation in intensive care. N Engl J Med 2012 Nov;367(20):1901-1911.

15. Van Der Linden P, James M, Mythen M, Weiskopf RB. Safety of modern starches used during surgery. Anesth Analg 2013 Jan;116(1):35-48.

16. Carless PA, Henry DA, Moxey AJ, O'Connell D, Brown T, Fergusson DA. Cell salvage for minimising perioperative allogeneic blood transfusion. Cochrane Database Syst Rev 2010;(4):CD001888. 